\title{
ON REFLEXIVE NORMS FOR THE DIRECT PRODUCT OF BANACH SPACES
}

\author{
BY \\ ROBERT SCHATTEN
}

Introduction. In a previous paper [7](1), for two Banach spaces $E_{1}, E_{2}$, the Banach spaces $E_{1} \otimes E_{2}, E_{1}^{\prime} \otimes E_{2}^{\prime}, E_{1}^{\prime \prime} \otimes E_{2}^{\prime \prime}[7$, p. 205] are constructed. If the norm $N$ [7, Definition 3.1] is defined on $E_{1} \otimes E_{2}$, then the associate norm $N^{\prime}$ [7, Definition 3.2 and Lemma 3.1] is defined on $E_{1}^{\prime} \otimes E_{2}^{\prime}$. Similarly $N^{\prime \prime}$ denotes the norm on $E_{1}^{\prime \prime} \otimes E_{2}^{\prime \prime}$.

Among the unsolved problems (mentioned in $[7, \S 6]$ ), are listed the following two:

A. What are the exact conditions imposed upon a crossnorm [7, Definition 3.3] under which $\left(E_{1} \otimes E_{2}\right)^{\prime}=E_{1}^{\prime} \otimes E_{2}^{\prime}$ holds?

$B$. Is the associate with every crossnorm also a crossnorm, or do there exist crossnorms whose associates are not crossnorms?

In the present paper we present a "partial" answer to problem A (which we denote by $A^{*}$ ), and a "partial" answer to problem $B$ (which we denote by $B^{*}$ ).

$\mathrm{A}^{*}$. A uniformly convex crossnorm $N$ sets up the relation $\left(E_{1} \otimes E_{2}\right)^{\prime}$ $=E_{1}^{\prime} \otimes E_{2}^{\prime}$ if, and only if, $N^{\prime \prime}=N$.

$\mathrm{B}^{*}$. For reflexive Banach spaces (that is, such that $E_{1}^{\prime \prime}=E_{1}, E_{2}^{\prime \prime}=E_{2}$ ) the associate with every crossnorm is also a crossnorm.

In this paper we also show that the values of a crossnorm for all expressions of rank not greater than 2 do not necessarily determine the crossnorm.

The following should be mentioned in immediate connection with problem A:

It is evident that for norms for which $\left(E_{1} \otimes E_{2}\right)^{\prime}=E_{1}^{\prime} \otimes E_{2}^{\prime}$ holds, $N^{\prime \prime}=N$. Since in general (for any norm $N$ ) all we can state is $\left(E_{1} \otimes E_{2}\right)^{\prime} \supset E_{1}^{\prime} \otimes E_{2}^{\prime}$ $\left[7\right.$, p. 205], we have no basis for assuming that $N^{\prime \prime}$ represents the norm in $\left(E_{1} \otimes E_{2}\right)^{\prime \prime}$, or $N^{\prime \prime}=N$ for expressions in $\mathscr{A}\left(E_{1}, E_{2}\right) \subset \mathfrak{A}\left(E_{1}^{\prime \prime}, E_{2}^{\prime \prime}\right)$ [7, Definition 1.3]. Therefore, $N^{\prime \prime} \leqq N[7$, Lemma 3.2] is the best that can be stated in the general case.

In the present paper we present some results on reflexive norms, that is, such that $N^{\prime \prime}=N$.

Presented to the Society, April 24, 1943, under the title "On certain properties of crossnorms." Received by the editors March 11, 1943, and, in revised form, June 3, 1943.

(1) Numerals in brackets refer to the bibliography at the end of this paper. Throughout this paper we shall use the results and notation of [7], with a slight modification. Since the printer does not find it easy to handle double and triple bars over expressions by machines, we shall write $E^{\prime}$ instead of $\bar{E}, E^{\prime \prime}$ instead of $\vec{E}, E^{\prime \prime \prime}$ instead of $\overline{\bar{E}}$. Similarly $N^{\prime}$ shall take the place of $\bar{N}$, and $N^{\prime \prime}$ that of $\bar{N}$. Thus $\left\langle N^{\prime \prime}\right\rangle^{\prime}$ shall stand for $\left.\overline{\overline{\langle}}\right\rangle$. 
- In $\$ 1$ we prove that norms that are reflexive, minimal, or have an associate property are identical. In $\$ 2$ we show that for reflexive Banach spaces the associate with every crossnorm is also a crossnorm, that is, there exists a least crossnorm. In $\$ 3$ we present a method for construction of reflexive crossnorms and prove certain inequalities. An application of the results of this section to Hilbert spaces permits us to construct semi-self-associate crossnorms, that is, for every natural number $k$ a crossnorm $S_{k}$ is constructed such that $S_{k} \neq S$ and $S_{k}=\left(S_{k}\right)^{\prime}=S$ for all expressions of rank not greater than $k$, where $S$ denotes the self-associate crossnorm for Hilbert spaces constructed by F. J. Murray and J. v. Neumann [5]. This last result also proves that a crossnorm is not determined by the values which it assumes for all expressions of rank not greater than 2. Finally, in $\$ 4$ we show that a uniformly convex crossnorm sets up the relation $\left(E_{1} \otimes E_{2}\right)^{\prime}=E_{1}^{\prime} \otimes E_{2}^{\prime}$ if, and only if, $N^{\prime \prime}=N$.

1. In this section we shall assume that $E_{1}, E_{2}$ are Banach spaces, with no special restrictions.

We introduce the following additional notation:

a. If a norm $N$ is defined on $\mathfrak{A}\left(E_{1}, E_{2}\right)$, then $N^{\prime \prime}$ is defined on $\mathfrak{A}\left(E_{1}^{\prime \prime}, E_{2}^{\prime \prime}\right)$ $\supset \mathfrak{A}\left(E_{1}, E_{2}\right)$. By $\left\langle N^{\prime \prime}\right\rangle$ we shall understand $N^{\prime \prime}$ considered only on $\mathfrak{A}\left(E_{1}, E_{2}\right)$. Similarly if $N$ is defined on $\mathfrak{A}\left(E_{1}, E_{2}\right), N^{\prime \prime \prime}$ is defined on $\mathfrak{A}\left(E_{1}^{\prime \prime \prime}, E_{2}^{\prime \prime \prime}\right)$ $\supset \mathfrak{A}\left(E_{1}^{\prime}, E_{2}^{\prime}\right)$, and $\left\langle N^{\prime \prime \prime}\right\rangle$ denotes $N^{\prime \prime \prime}$ considered only on $\mathfrak{A}\left(E_{1}^{\prime}, E_{2}^{\prime}\right)$.

b. The set $\mathfrak{A}\left(E_{1}, E_{2}\right)$ in which there is defined a norm $N$, we shall denote by $\mathfrak{A}_{N}\left(E_{1}, E_{2}\right)$. Thus $\mathfrak{A}_{N^{\prime}}\left(E_{1}^{\prime}, E_{2}^{\prime}\right)$ shall denote the set $\mathfrak{A}\left(E_{1}^{\prime}, E_{2}^{\prime}\right)$ in which there is defined the norm $N^{\prime}$ associate with $N$.

c. The symbol $\vdots \bar{f} \in \mathfrak{A}^{*}\left(E_{1}, E_{2}\right) \vdots \sup |\bar{F}(\bar{f})| / N(\bar{f})$ shall denote the least upper bound for all numbers $|\bar{F}(\bar{f})| / N(\bar{f})$, obtained when $\bar{f}$ varies over $\mathfrak{Q} *\left(E_{1}, E_{2}\right)$.

Lemma 1.1. If $N$ is a norm in $\mathfrak{A}\left(E_{1}, E_{2}\right)$, then $N^{\prime}=\left\langle N^{\prime \prime}\right\rangle^{\prime}=N^{\prime \prime \prime}$ for $\bar{F}$ in $\mathfrak{U}^{*}\left(E_{1}^{\prime}, E_{2}^{\prime}\right) \subset \mathfrak{U}^{*}\left(E_{1}^{\prime \prime \prime}, E_{2}^{\prime \prime \prime}\right)$.

Proof. Let $\bar{F} \in \mathfrak{A}^{*}\left(E_{1}^{\prime}, E_{2}^{\prime}\right)$. Then

$$
\begin{aligned}
N^{\prime}(\bar{F}) & =\vdots \bar{f} \in \mathfrak{A}^{*}\left(E_{1}, E_{2}\right) \vdots \sup |\bar{F}(\bar{f})| / N(\bar{f}) \\
& \leqq \vdots \bar{f} \in \mathfrak{A}^{*}\left(E_{1}, E_{2}\right) \vdots \sup |\bar{F}(\bar{f})| / N^{\prime \prime}(\bar{f})=\left\langle N^{\prime \prime}\right\rangle^{\prime}(\bar{F}) \\
& \leqq \vdots \bar{f} \in \mathfrak{A}^{*}\left(E_{1}^{\prime \prime}, E_{2}^{\prime \prime}\right) \vdots \sup |\bar{F}(\bar{f})| / N^{\prime \prime}(\bar{f})=N^{\prime \prime \prime}(\bar{F}) .
\end{aligned}
$$

On the other hand, $N^{\prime \prime \prime}(\bar{F}) \leqq N^{\prime}(\bar{F})$ [7, Lemma 3.2]. This completes the proof.

Definition 1.1. A norm $N$ will be termed minimal if, for every norm $N^{0}$ for which $N^{\prime}=\left(N^{0}\right)^{\prime}$, we have $N^{0} \geqq N$.

Definition 1.2. A norm $N$ will be termed reflexive if $\left\langle N^{\prime \prime}\right\rangle=N$.

Definition 1.3. A norm $N$ in $\mathfrak{A}\left(E_{1}, E_{2}\right)$ will be said to have an "associate 
property" if, for a certain norm $N^{0}$ in $\mathfrak{A}\left(E_{1}^{\prime}, E_{2}^{\prime}\right), \mathfrak{A}_{N}\left(E_{1}, E_{2}\right) \subset \mathfrak{A}_{\left(N^{0}\right)},\left(E_{1}^{\prime \prime}, E_{2}^{\prime \prime}\right)$.

THEOREM 1.1. For a norm $N$, the following statements are equivalent:

(a) $N$ is minimal,

(b) $N$ is reflexive,

(c) $N$ has an associate property.

Proof. We shall prove (a) $\rightarrow$ (b) $\rightarrow$ (c) $\rightarrow$ (a). Let $N$ be minimal. By Lemma 1.1, $\left\langle N^{\prime \prime}\right\rangle^{\prime}=N^{\prime}$ for $\bar{F} \in \mathfrak{I}^{*}\left(E_{1}^{\prime}, E_{2}^{\prime}\right)$ and any norm $N$ in $\mathfrak{A}\left(E_{1}, E_{2}\right)$. Thus $\left\langle N^{\prime \prime}\right\rangle$ and $N$ have the same associate. Since $N$ is minimal by hypothesis, this means $\left\langle N^{\prime \prime}\right\rangle \geqq N$. On the other hand, for any norm $N,\left\langle N^{\prime \prime}\right\rangle \leqq N[7$, Lemma 3.2]. Thus $\left\langle N^{\prime \prime}\right\rangle=N$. We have proved (a) $\rightarrow$ (b).

Suppose $N$ is reflexive, that is, $\left\langle N^{\prime \prime}\right\rangle=N$. Then obviously $N$ has an associate property, because

$$
\mathfrak{A}_{N}\left(E_{1}, E_{2}\right)=\mathfrak{A}_{\left\langle N^{\prime \prime}\right\rangle}\left(E_{1}, E_{2}\right) \subset \mathfrak{A}_{N^{\prime \prime}}\left(E_{1}^{\prime \prime}, E_{2}^{\prime \prime}\right) .
$$

Therefore (b) $\rightarrow$ (c).

Suppose finally that $N$ defined in $\mathfrak{A}\left(E_{1}, E_{2}\right)$ has an associate property, that is, for a certain norm $N^{0}$ defined in $\mathfrak{A}\left(E_{1}^{\prime}, E_{2}^{\prime}\right), \mathfrak{A}_{N}\left(E_{1}, E_{2}\right) \subset \mathfrak{A}_{\left(N^{0}\right)},\left(E_{1}^{\prime \prime}, E_{2}^{\prime \prime}\right)$. Let the norm $N^{00}$ in $\mathfrak{A}\left(E_{1}, E_{2}\right)$ be such that $\left(N^{00}\right)^{\prime}=N^{\prime}$. Then, for $\bar{F} \in \mathfrak{A}^{*}\left(E_{1}^{\prime}, E_{2}^{\prime}\right)$, we have

$$
\begin{aligned}
\left(N^{00}\right)^{\prime}(\bar{F})=N^{\prime}(\bar{F}) & =\vdots \bar{f} \in \mathfrak{A}^{*}\left(E_{1}, E_{2}\right) \vdots \sup |\bar{F}(\bar{f})| / N(\bar{f}) \\
& \leqq \vdots \bar{f} \in \mathfrak{A}^{*}\left(E_{1}^{\prime \prime}, E_{2}^{\prime \prime}\right) \vdots \sup |\bar{F}(\bar{f})| /\left(N^{0}\right)^{\prime}(\bar{f}) \\
& =\left(N^{0}\right)^{\prime \prime}(\bar{F}) \leqq N^{0}(\bar{F}) .
\end{aligned}
$$

Thus, $\left(N^{00}\right)^{\prime} \leqq N^{0}$ throughout $\mathfrak{A}\left(E_{1}^{\prime}, E_{2}^{\prime}\right)$, and therefore $\left(N^{00}\right)^{\prime \prime} \geqq\left(N^{0}\right)^{\prime}$ throughout $\mathscr{A}\left(E_{1}^{\prime \prime}, E_{2}^{\prime \prime}\right)$. Consequently, $N^{00} \geqq\left(N^{00}\right)^{\prime \prime} \geqq\left(N^{0}\right)^{\prime}=N$, for $\bar{f}$ in $\mathfrak{A}^{*}\left(E_{1}, E_{2}\right) \subset \mathfrak{A}^{*}\left(E_{1}^{\prime \prime}, E_{2}^{\prime \prime}\right)$. Thus $N$ is minimal. Therefore (c) $\rightarrow$ (a). This completes the proof.

Corolla RY 1. If $N$ and $N^{0}$ denote two reflexive norms, such that $N \leqq N^{0}$ and $N \neq N^{0}$, then $N^{\prime} \geqq\left(N^{0}\right)^{\prime}$ and $N^{\prime} \neq\left(N^{0}\right)^{\prime}$.

Proof. Obviously $N^{\prime} \geqq\left(N^{0}\right)^{\prime}$. If it were $N^{\prime}=\left(N^{0}\right)^{\prime}$, then $N^{\prime \prime}=\left(N^{0}\right)^{\prime \prime}$ and $\left\langle N^{\prime \prime}\right\rangle=\left\langle\left(N^{0}\right)^{\prime \prime}\right\rangle$. By hypothesis $N$ and $N^{0}$ are reflexive. Thus Definition 1.2 gives $N=N^{0}$. This contradicts our assumption.

Corollary 2. If $N$ and $N^{0}$ denote two norms in $\mathfrak{A}\left(E_{1}, E_{2}\right)$, of which $N$ is reflexive, and $N \leqq N^{0}$ throughout $\mathfrak{Q}\left(E_{1}, E_{2}\right)$, then $N \leqq\left\langle\left(N^{0}\right)^{\prime \prime}\right\rangle$.

Proof. By assumption, $N \leqq N^{0}$. Therefore $N^{\prime} \geqq\left(N^{0}\right)^{\prime}$ and $N^{\prime \prime} \leqq\left(N^{0}\right)^{\prime \prime}$ [7, Lemma 3.3]. Thus $\left\langle N^{\prime \prime}\right\rangle \leqq\left\langle\left(N^{0}\right)^{\prime \prime}\right\rangle . N$ is reflexive by assumption. Therefore $N=\left\langle N^{\prime \prime}\right\rangle$, and $N \leqq\left\langle\left(N^{0}\right)^{\prime \prime}\right\rangle$. This completes the proof.

Remark. Property (c) of Theorem 1.1 suggests the existence of an infinite number of different reflexive crossnorms. We shall prove later that this is 
the case, and present a method for construction of reflexive crossnorms $\left({ }^{2}\right)$.

2. Throughout the rest of this paper we shall assume $E_{1}=E_{1}^{\prime \prime}, E_{2}=E_{2}^{\prime \prime}$. In this case $\mathfrak{A}\left(E_{1}, E_{2}\right)=\mathfrak{A}\left(E_{1}^{\prime \prime}, E_{2}^{\prime \prime}\right)$, and for any norm $N$ in $\mathfrak{A}\left(E_{1}, E_{2}\right)$, $\left\langle N^{\prime \prime}\right\rangle=N^{\prime \prime},\left\langle N^{\prime \prime \prime}\right\rangle=N^{\prime \prime \prime}$. It should be noticed, however, that many results of the following sections are also valid for general Banach spaces.

Let $N_{L}\left(E_{1}, E_{2}\right), N_{G}\left(E_{1}, E_{2}\right)$ denote the least crossnorm whose associate is also a crossnorm and the greatest crossnorm in $\mathfrak{A}\left(E_{1}, E_{2}\right)$, respectively; in the case where there is no fear of misunderstanding, we shall write simply $N_{L}$, $N_{G}$ [7, Definitions 4.1, 4.2, Lemmas 4.1, 4.2 and Theorem 4.1].

Lemma 2.1. $N_{L}\left(E_{1}, E_{2}\right)=N_{L}\left(E_{1}^{\prime \prime}, E_{2}^{\prime \prime}\right)$ and $N_{G}\left(E_{1}, E_{2}\right)=N_{G}\left(E_{1}^{\prime \prime}, E_{2}^{\prime \prime}\right)$.

Proof. This is a consequence of the definition of the least and greatest crossnorms [7, Definitions 4.1, 4.2].

Lemma 2.2 For any crossnorm $N$ in $\mathfrak{A}\left(E_{1}, E_{2}\right)$ for which $N \geqq N_{L}\left(E_{1}, E_{2}\right)$, we have $N^{\prime} \geqq N_{L}\left(E_{1}^{\prime}, E_{2}^{\prime}\right), N^{\prime \prime} \geqq N_{L}\left(E_{1}^{\prime \prime}, E_{2}^{\prime \prime}\right)=N_{L}\left(E_{1}, E_{2}\right), \cdots$.

Proof. Let $N$ denote a crossnorm whose associate is also a crossnorm, that is, $N \geqq N_{L}$ [7, Theorem 4.1]. Since $N^{\prime \prime} \leqq N$ [7, Lemma 3.2], $N^{\prime \prime}(f \otimes \varphi)$ $\leqq N(f \otimes \varphi)=\|f\|\|\varphi\|$ for $f \in E_{1}, \varphi \in E_{2}$. But $N^{\prime \prime}$ is the associate of the crossnorm $N^{\prime}$. Therefore $N^{\prime \prime}(f \otimes \varphi) \geqq\|f\|\|\varphi\|\left[7\right.$, Lemma 4.3]. Thus $N^{\prime \prime}$ is a crossnorm, or the associate with the crossnorm $N^{\prime}$ in $\mathfrak{A}\left(E_{1}^{\prime}, E_{2}^{\prime}\right)$ is a crossnorm. This gives $N^{\prime} \geqq N_{L}\left(E_{1}^{\prime}, E_{2}^{\prime}\right)$ [7, Theorem 4.1]. From $N \geqq N_{L}\left(E_{1}, E_{2}\right)$ we conclude $N^{\prime} \geqq N_{L}\left(E_{1}^{\prime}, E_{2}^{\prime}\right)$. Similarly from $N^{\prime} \geqq N_{L}\left(E_{1}^{\prime}, E_{2}^{\prime}\right)$ we conclude $N^{\prime \prime} \geqq N_{L}\left(E_{1}^{\prime \prime}, E_{2}^{\prime \prime}\right)=N_{L}\left(E_{1}, E_{2}\right)$, by Lemma 2.1 . This completes the proof.

LEMMA 2.3. $N_{L}$ is reflexive.

Proof. Obviously $N_{L} \geqq N_{L}$. Therefore $\left(N_{L}\right)^{\prime \prime} \geqq N_{L}$, by virtue of Lemma 2.2. But $\left(N_{L}\right)^{\prime \prime} \leqq N_{L}\left[7\right.$, Lemma 3.2]. Therefore $\left(N_{L}\right)^{\prime \prime}=N_{L}$.

LEMMA 2.4. The associate with the greatest crossnorm is the least crossnorm, that is, $\left(N_{G}\left(E_{1}, E_{2}\right)\right)^{\prime}=N_{L}\left(E_{1}^{\prime}, E_{2}^{\prime}\right)$.

Proof. Since $N_{G}\left(E_{1}, E_{2}\right) \geqq N_{L}\left(E_{1}, E_{2}\right),\left(N_{G}\left(E_{1}, E_{2}\right)\right)^{\prime} \geqq N_{L}\left(E_{1}^{\prime}, E_{2}^{\prime}\right)$ as follows from Lemma 2.2. But $\left(N_{L}\left(E_{1}^{\prime}, E_{2}^{\prime}\right)\right)^{\prime}$ is a crossnorm [7, Theorem 4.1]. Thus $\left(N_{L}\left(E_{1}^{\prime}, E_{2}^{\prime}\right)\right)^{\prime} \leqq N_{G}\left(E_{1}^{\prime \prime}, E_{2}^{\prime \prime}\right)=N_{G}\left(E_{1}, E_{2}\right)$ [7, Theorem 4.2], or $N_{L}\left(E_{1}^{\prime}, E_{2}^{\prime}\right)=\left(N_{L}\left(E_{1}^{\prime}, E_{2}^{\prime}\right)\right)^{\prime \prime} \geqq\left(N_{G}\left(E_{1}, E_{2}\right)\right)^{\prime}$, as follows from Lemma 2.3. Therefore $\left(N_{G}\left(E_{1}, E_{2}\right)\right)^{\prime}=N_{L}\left(E_{1}^{\prime}, E_{2}^{\prime}\right)$. This completes the proof.

Corollary. $N_{L}$ and $N_{G}^{\prime \prime}$ are associate with each other.

Proof. The associate with $N_{G}^{\prime \prime}$ is $N_{G}^{\prime \prime \prime}=N_{G}^{\prime}=N_{L}$, by Lemmas 2.4 and 1.1.

(2) The question of existence of non-reflexive crossnorms is not settled in this paper. It should be noticed that for every non-reflexive norm, $E_{1}^{\prime} \otimes E_{2}^{\prime}$ would be a proper subset of $\left(E_{1} \otimes E_{2}\right)^{\prime}$. In particular it is not settled whether the greatest crossnorm is reflexive. It can be shown, however, that when $E_{1}, E_{2}$ are Hilbert spaces, then the greatest crossnorm is reflexive. 
The associate with $N_{L}$ is $N_{L}^{\prime}=N_{G^{\prime}}$, by Lemma 2.4 .

LEMma 2.5. If, for a certain crossnorm $N, N^{\prime} \geqq N_{L}$, then $N \geqq N_{L}$.

Proof. Lemma 2.2 gives $N^{\prime \prime} \geqq N_{L}$, therefore $N \geqq N^{\prime \prime} \geqq N_{L}$. This completes the proof.

THEOREM 2.1. Every crossnorm $N \geqq N_{L}$.

Proof. Since $N$ is a crossnorm, $N \leqq N_{G}$ [7, Lemma 4.2]. Therefore $N^{\prime} \geqq N_{G}^{\prime}=N_{L}$, as follows from Lemma 2.4. Thus Lemma 2.5 gives $N \geqq N_{L}$. This completes the proof.

THEOREM 2.2. The associate with every crossnorm is also a crossnorm.

Proof. This is a consequence of Theorem 2.1 and [7, Theorem 4.1].

THEOREM 2.3. There exists a least crossnorm.

Proof. It is obviously $N_{L}$, as follows from Theorem 2.1 and [7, Definition 4.2 and Lemma 4.4].

Corollary. For every crossnorm $N$ in $\mathfrak{A}\left(E_{1}, E_{2}\right)$,

$$
\left\|\sum_{i=1}^{n} F\left(f_{i}\right) \varphi_{i}\right\| \leqq\|F\| N\left(\sum_{i=1}^{n} f_{i} \otimes \varphi_{i}\right) \quad \text { for } \bar{F} \in E_{1} .
$$

Proof. This is a consequence of Theorem 2.1 and [7, Theorem 4.1.1].

The question of existence of a norm not less than $N_{L}$ which is not a crossnorm, and whose associate is a crossnorm, remains open. It is clear that if such a norm exists, it must be non-reflexive.

3. Definition 3.1. Let $E_{1}, E_{2}$ denote two Banach spaces, and $N$ a crossnorm in $\mathfrak{A}\left(E_{1}^{\prime}, E_{2}^{\prime}\right)$. We define a sequence of functions $\left\{N_{k}\right\}$ for expressions in $\mathfrak{A}\left(E_{1}, E_{2}\right)=\mathfrak{A}\left(E_{1}^{\prime \prime}, E_{2}^{\prime \prime}\right)$ in the following way:

$$
N_{k}\left(\sum_{i=1}^{n} f_{i} \otimes \varphi_{i}\right)=\sup \left|\left(\sum_{j=1}^{k} F_{j} \otimes \phi_{j}\right)\left(\sum_{i=1}^{n} f_{i} \otimes \varphi_{i}\right)\right| / N\left(\sum_{j=1}^{k} F_{j} \otimes \phi_{j}\right)
$$

where sup, that is, the least upper bound, is taken for all sequences of $k$ terms $F_{1}, \cdots, F_{k}$ in $E_{1}^{\prime}$ and $\phi_{1}, \cdots, \phi_{k}$ in $E_{2}^{\prime}$.

THEOREM 3.1. For every natural $k, N_{k}$ is a crossnorm in $\mathfrak{A}\left(E_{1}, E_{2}\right)$.

Proof. The proof is similar to that of [7, Theorem 7.2].

From the definition, $N_{L}=N_{1} \leqq N_{2} \leqq N_{3} \leqq \cdots$ and $\lim _{k \rightarrow \infty} N_{k}=N^{\prime}$.

THEOREM 3.2. For every natural $k$, the crossnorm $N_{k}$ is reflexive.

Proof. Suppose that for a certain expression $\sum_{i=1}^{s} f_{i}^{0} \otimes \varphi_{i}^{0} \in \mathfrak{A}\left(E_{1}, E_{2}\right)$, $N_{k}^{\prime \prime}\left(\sum_{i=1}^{s} f_{i}^{0} \otimes \varphi_{i}^{0}\right)<N_{k}\left(\sum_{i=1}^{s} f_{i}^{0} \otimes \varphi_{i}^{0}\right)-\epsilon$, where $\epsilon$ is a certain positive number. 
Then for a given expression $\sum_{j=1}^{m} F_{j} \otimes \phi_{j}$ in $\mathfrak{A}\left(E_{1}^{\prime}, E_{2}^{\prime}\right)$, there exists an expres$\operatorname{sion} \sum_{i=1}^{n} f_{i} \otimes \varphi_{i}$, such that

$$
\begin{aligned}
& \left|\left(\sum_{j=1}^{m} F_{j} \otimes \phi_{j}\right)\left(\sum_{i=1}^{8} f_{i}^{0} \otimes \varphi_{i}^{0}\right)\right| N_{k}\left(\sum_{i=1}^{n} f_{i} \otimes \varphi_{i}\right) \\
& \left|\left(\sum_{j=1}^{m} F_{j} \otimes \phi_{j}\right)\left(\sum_{i=1}^{n} f_{i} \otimes \varphi_{i}\right)\right| \\
& <N_{k}\left(\sum_{i=1}^{8} f_{i}^{0} \otimes \varphi_{i}^{0}\right)-\epsilon .
\end{aligned}
$$

This means

$$
\begin{array}{r}
\left|\left(\sum_{j=1}^{m} F_{i} \otimes \phi_{j}\right)\left(\sum_{i=1}^{s} f_{i}^{0} \otimes \varphi_{i}^{0}\right)\right|\left|\left(\sum_{j=1}^{k} \hat{F}_{j} \otimes \hat{\phi}_{j}\right)\left(\sum_{i=1}^{n} f_{i} \otimes \varphi_{i}\right)\right| \\
\left|\left(\sum_{i=1}^{m} F_{j} \otimes \phi_{j}\right)\left(\sum_{i=1}^{n} f_{i} \otimes \varphi_{i}\right)\right| N\left(\sum_{j=1}^{k} \hat{F}_{j} \otimes \hat{\phi}_{j}\right) \\
<N_{k}\left(\sum_{i=1}^{s} \hat{f}_{i}^{0} \otimes \varphi_{i}^{0}\right)-\epsilon
\end{array}
$$

for every sequence of $k$ terms $\hat{F}_{1}, \cdots, \hat{F}_{k}$ in $E_{1}^{\prime} ; \hat{\phi}_{1}, \cdots, \hat{\phi}_{k}$ in $E_{2}^{\prime}$. Taking originally for $\sum_{j=1}^{m} F_{j} \otimes \phi_{j}$ an expression of the form $\sum_{j=1}^{k} \hat{\mathrm{F}}_{j} \otimes \hat{\phi}_{j}$ we get $\left|\left(\sum_{j=1}^{k} \hat{F}_{j} \otimes \hat{\phi}_{j}\right)\left(\sum_{i=1}^{s} f_{i}^{0} \otimes \varphi_{i}^{0}\right)\right| / N\left(\sum_{j=1}^{k} \hat{F}_{j} \otimes \hat{\phi}_{j}\right)<N_{k}\left(\sum_{i=1}^{s} f_{i}^{0} \otimes \varphi_{i}^{0}\right)-\epsilon$ for every sequence of $k$ terms $\hat{\mathrm{F}}_{1}, \cdots, \hat{F}_{k}$ in $E_{1}^{\prime}, \hat{\phi}_{1}, \cdots, \hat{\phi}_{k}$ in $E_{2}^{\prime}$. Therefore $N_{k}\left(\sum_{i=1}^{s} f_{i}^{0} \otimes \varphi_{i}^{0}\right) \leqq N_{k}\left(\sum_{i=1}^{s} f_{i}^{0} \otimes \varphi_{i}^{0}\right)-\epsilon$, as follows from Definition 3.1. The last inequality cannot hold. This completes the proof.

THEOREM 3.3. If $N$ is a reflexive crossnorm, then $\left(N_{k}\right)^{\prime}=N$ for all expressions of rank not greater than $k$.

Proof. Obviously $\left(N_{k}\right)^{\prime} \leqq N$ for all expressions of rank not greater than $k$ as a consequence of Definition 3.1 for $N_{k}$, and that of an associate with a given norm. On the other hand $N_{k} \leqq N^{\prime}$ everywhere in $\mathfrak{A}\left(E_{1}, E_{2}\right)$. Therefore $\left(N_{k}\right)^{\prime} \geqq N^{\prime \prime}=N$. This completes the proof.

THEOREM 3.4. If $N$ is a reflexive crossnorm, then $N_{k}$ is the least crossnorm whose associate equals $N$ for all expressions of rank not greater than $k$.

Proof. Theorem 3.3 gives $\left(N_{k}\right)^{\prime}=N$ for all expressions of rank not greater than $k$. On the other hand it is easy to see that if a crossnorm $N^{0}$ satisfies the inequality $N^{0}\left(\sum_{i=1}^{n} f_{i} \otimes \varphi_{i}\right)<N_{k}\left(\sum_{i=1}^{n} f_{i} \otimes \varphi_{i}\right)$ for a certain expression $\sum_{i=1}^{n} f_{i} \otimes \varphi_{i}$, then there exists an expression $\sum_{j=1}^{k} F_{j} \otimes \phi_{j}$, such that $\left(N^{0}\right)^{\prime}\left(\sum_{j=1}^{k} F_{j} \otimes \phi_{j}\right)$ $>N\left(\sum_{j=1}^{k} F_{j} \otimes \phi_{j}\right)$. Thus $\left(N^{0}\right)^{\prime}=N$, for all expressions of rank not greater than $k$, implies $N^{0} \geqq N_{k}$. This completes the proof.

It is not difficult to see that the last theorem is a generalization of [7, Theorem 4.1]. 
In the case $E_{1}$ and $E_{2}$ are Hilbert spaces, we may assume $\mathfrak{A}\left(E_{1}, E_{2}\right)$ $=\mathfrak{A}\left(E_{1}^{\prime}, E_{2}^{\prime}\right)$. In this case, $N$ and $N^{\prime}$ are defined in $\mathfrak{A}\left(E_{1}, E_{2}\right)$. For the case of Hilbert spaces, F. J. Murray and J. v. Neumann define in $\mathfrak{A}\left(E_{1}, E_{2}\right)$ a selfassociate crossnorm, which we shall denote by $S$ [5].

TheOREM 3.5. For every natural $p, S_{p}=\left(S_{p}\right)^{\prime}=S$ for all expressions of rank not greater than $p$.

Proof. Since $S=S^{\prime}, S=S^{\prime \prime}$. Thus $S$ is reflexive. Theorem 3.3 gives $\left(S_{p}\right)^{\prime}=S$ for all expressions of rank not greater than $p$. Now suppose that for a certain expression $\sum_{i=1}^{n} f_{i} \otimes \varphi_{i}$ and a certain crossnorm $N \leqq S$, $N^{\prime}\left(\sum_{i=1}^{n} f_{i} \otimes \varphi_{i}\right)=S\left(\sum_{i=1}^{n} f_{i} \otimes \varphi_{i}\right)$. Since $N \leqq S, N^{\prime} \geqq S^{\prime}=S$. But $S^{2} \leqq N N^{\prime}$ [7, p. 213]. Therefore $N\left(\sum_{i=1}^{n} f_{i} \otimes \varphi_{i}\right)=S\left(\sum_{i=1}^{n} f_{i} \otimes \varphi_{i}\right)$. An application of the last remark to the fact that $S_{p} \leqq S$ and $\left(S_{p}\right)^{\prime}=S$, for all expressions of rank not greater than $p$, gives $S_{p}=S$ for all expressions of rank not greater than $p$. Thus $S_{p}=\left(S_{p}\right)^{\prime}=S$ for all expressions of rank not greater than $p$. This completes the proof.

THEOREM 3.6. For every natural $p, S_{p} \neq S_{p+1}$.

Proof. That $S_{2} \neq S_{3}\left({ }^{3}\right)$ is shown in the following manner: Consider an expression $f_{1} \otimes \varphi_{1} \cdot+\cdot f_{2} \otimes \varphi_{2} \cdot+\cdot f_{3} \otimes \varphi_{3}$, where $f_{1}, f_{2}, f_{3}$, and $\varphi_{1}, \varphi_{2}, \varphi_{3}$ form orthonormal sets. A calculation shows that

$$
\begin{aligned}
S_{2}\left(f_{1} \otimes \varphi_{1} \cdot\right. & \left.+\cdot f_{2} \otimes \varphi_{2} \cdot+\cdot f_{3} \otimes \varphi_{3}\right) \\
& =\sup \frac{\mid\left(h_{1} \otimes \chi_{1} \cdot+\cdot h_{2} \otimes \chi_{2}\right)\left(f_{1} \otimes \varphi_{1} \cdot+f_{2} \otimes \varphi_{2} \cdot+\cdot f_{3} \otimes \varphi_{3}\right)}{S\left(h_{1} \otimes \chi_{1} \cdot+\cdot h_{2} \otimes \chi_{2}\right)}
\end{aligned}
$$

where sup, that is, the least upper bound, is taken for all pairs $h_{1}, h_{2}$, in $\mathfrak{M}_{1}$ (the closed linear manifold determined by $f_{1}, f_{2}, f_{3}$ ) and $\chi_{1}, \chi_{2}$, in $\mathfrak{M}_{2}$ (the closed linear manifold determined by $\left.\varphi_{1}, \varphi_{2}, \varphi_{3}\right)$. From this it is easily concluded that $S_{2}\left(f_{1} \otimes \varphi_{1} \cdot+\cdot f_{2} \otimes \varphi_{2} \cdot+\cdot f_{3} \otimes \varphi_{3}\right) \leqq 2^{1 / 2}$ and

$$
S_{3}\left(f_{1} \otimes \varphi_{1} \cdot+\cdot f_{2} \otimes \varphi_{2} \cdot+\cdot f_{3} \otimes \varphi_{3}\right)=S\left(f_{1} \otimes \varphi_{1} \cdot+\cdot f_{2} \otimes \varphi_{2} \cdot+\cdot f_{3} \otimes \varphi_{3}\right)=3^{1 / 2}
$$

A similar reasoning can be applied to prove $S_{p} \neq S_{p+1}$ for any natural $p$. Corollary. $S_{p} \neq S$ for $p=1,2,3, \cdots$.

Proof. Obviously $S_{p} \leqq S_{p+1} \leqq S$. But $S_{p} \neq S_{p+1}$. Therefore $S_{p} \neq S$. This completes the proof $\left({ }^{4}\right)$.

(3) This result and proof is due to F. J. Murray.

(4) For a reflexive (or not) norm $N, N_{k}$ is reflexive. There exist, however, reflexive norms, for instance $S$, such that for no reflexive norm $N$ is $S=N_{k}$. Proof. For a reflexive $N$, Definition 3.1 and Theorem 3.3 give $\left(\left(N_{k}\right)^{\prime}\right)_{k}=N_{k}$. If it were $S=N_{k}$ for a reflexive $N$, then $\left(N_{k}\right)^{\prime}=S^{\prime}=S$ and $S_{k}=S$ as a consequence of the preceding relation. We have shown above that this is not the case. This completes the proof. 
Remark. From Theorems 3.5 and 3.6 it is evident that we have constructed three different reflexive crossnorms, which are equal for all expressions of rank not greater than $p$; namely $S_{p},\left(S_{p}\right)^{\prime}, S$.

$S_{p}$ and $\left(S_{p}\right)^{\prime}$ are reflexive, hence associate with each other.

Thus for every natural $p$ we have constructed reflexive "semi-self-associate" crossnorms $S_{p}$ and $\left(S_{p}\right)^{\prime}$, that is not self-associate, but equal to their associates for all expressions of rank not greater than $p$.

Incidentally, this result also proves that the values of a crossnorm for all expressions of rank not greater than $p$ (where $p$ denotes any natural number) do not necessarily determine the crossnorm.

4. In the introduction of this paper, it was pointed out that if a norm sets up the relation $\left(E_{1} \otimes E_{2}\right)^{\prime}=E_{1}^{\prime} \otimes E_{2}^{\prime}$, then $N^{\prime \prime}=N$ for expressions in $\mathfrak{A}\left(E_{1}, E_{2}\right) \subset \mathfrak{A}\left(E_{1}^{\prime \prime}, E_{2}^{\prime \prime}\right)$, or $N$ is reflexive. In the present section we consider a "converse" problem.

LemMA 4.1. If $N$ is a reflexive crossnorm in $\mathfrak{A}\left(E_{1}, E_{2}\right)$, then $E_{1} \otimes E_{2}$ $\subset E_{1}^{\prime \prime} \otimes E_{2}^{\prime \prime}$.

Proof. The linear set $\mathfrak{A}^{*}\left(E_{1}^{\prime \prime}, E_{2}^{\prime \prime}\right)$ in which there is defined the norm $N^{\prime \prime}$ is an extension of the linear set $\mathfrak{A}^{*}\left(E_{1}, E_{2}\right)$ in which there is defined the norm $N$. Thus the closure of $\mathfrak{Y}^{*}\left(E_{1}^{\prime \prime}, E_{2}^{\prime \prime}\right)$ is an extension of the closure of $\mathfrak{A}^{*}\left(E_{1}, E_{2}\right)$. This completes the proof.

Lemma 4.2. If $N$ is a reflexive crossnorm in $\mathfrak{X}\left(E_{1}, E_{2}\right)$, then $E_{1}^{\prime} \otimes E_{2}^{\prime}$ forms a fundamental subset of $\left(E_{1} \otimes E_{2}\right)^{\prime}$, that is if, for all $\bar{F}$ in $E_{1}^{\prime} \otimes E_{2}^{\prime}$ and a certain $\bar{f}_{0}$ in $E_{1} \otimes E_{2}, \bar{F}\left(\bar{f}_{0}\right)=0$, then $\bar{f}_{0}=0$.

Proof. For $\bar{F}_{0} \in E_{1}^{\prime} \otimes E_{2}^{\prime}$, we have $N^{\prime}\left(\bar{F}_{0}\right)=\sup \left|\bar{F}_{0}(\bar{f})\right| / N(\bar{f})$ where sup is taken over the set of all $\bar{f}^{\prime} s$ in $E_{1} \otimes E_{2}$ [7, Lemma 3.4]. Similarly, for $\bar{F}_{0}^{+} \in E_{1}^{\prime \prime} \otimes E_{2}^{\prime \prime}, N^{\prime \prime}\left(\bar{F}_{0}^{+}\right)=\sup \left|\bar{F}_{0}^{+}(\bar{F})\right| / N^{\prime}(\bar{F})$ where sup is taken over the set of all $\bar{F}^{\prime}$ 's in $E_{1}^{\prime} \otimes E_{2}^{\prime} . N$ is reflexive by assumption. Therefore for $\bar{f}_{0} \in E_{1} \otimes E_{2}$, Lemma 4.1 gives $N^{\prime \prime}\left(\bar{f}_{0}\right)=\sup \left|\bar{F}\left(\bar{f}_{0}\right)\right| / N^{\prime}(\bar{F})$, where sup is taken over the set of all $\bar{F}$ 's in $E_{1}^{\prime} \otimes E_{2}^{\prime}$. The second part of our assumption gives $N^{\prime \prime}\left(\bar{f}_{0}\right)=0$. But $N^{\prime \prime}=N$. Therefore $N\left(\bar{f}_{0}\right)=0$. Thus $\bar{f}_{0}=0$. This completes the proof.

LEMMA 4.3. If a reflexive crossnorm sets up the relation $\left(E_{1} \otimes E_{2}\right)^{\prime \prime}=E_{1} \otimes E_{2}$, then $\left(E_{1} \otimes E_{2}\right)^{\prime}=E_{1}^{\prime} \otimes E_{2}^{\prime}$.

Proof. Suppose that $\left(E_{1} \otimes E_{2}\right)^{\prime \prime}=E_{1} \otimes E_{2}$, and $\bar{F}^{*}$ is an element of $\left(E_{1} \otimes E_{2}\right)^{\prime}$ which does not belong to $E_{1}^{\prime} \otimes E_{2}^{\prime}$. From the construction of $E_{1}^{\prime} \otimes E_{2}^{\prime}$ follows that the set is closed in $\left(E_{1} \otimes E_{2}\right)^{\prime}$. Hence, there exists a linear functional $\mathcal{F}$ on $\left(E_{1} \otimes E_{2}\right)^{\prime}$, such that $\mathcal{F}\left(\bar{F}^{*}\right)=1$ and $\mathcal{F}(\bar{F})=0$, for $\bar{F}$ in $E_{1}^{\prime} \otimes E_{2}^{\prime}[1$, p. 57]. Since $\left(E_{1} \otimes E_{2}\right)^{\prime \prime}=E_{1} \otimes E_{2}$, there exists an $\bar{f}_{0}$ in $E_{1} \otimes E_{2}$, corresponding to $\mathcal{F}$, such that $\bar{F}^{*}\left(\bar{f}_{0}\right)=1$ and $\bar{F}\left(\bar{f}_{0}\right)=0$ for $\bar{F}$ in $E_{1}^{\prime} \otimes E_{2}^{\prime}$. The last condition im- 
plies $\bar{f}_{0}=0$ by Lemma 4.2 . This contradicts $\bar{F}^{*}\left(\bar{f}_{0}\right)=1$. This completes the proof.

THEOREM 4.1. If a reflexive crossnorm $N$ defined on $\mathfrak{A}\left(E_{1}, E_{2}\right)$ is uniformly convex [2], then $\left(E_{1} \otimes E_{2}\right)^{\prime}=E_{1}^{\prime} \otimes E_{2}^{\prime}$.

Proof. By continuity it follows that $N$ is uniformly convex in $E_{1} \otimes E_{2}$, hence $[3,4,6]\left(E_{1} \otimes E_{2}\right)^{\prime \prime}=E_{1} \otimes E_{2}$. An application of Lemma 4.3 completes the proof.

\section{REFERENCES}

1. S. Banach, Theorie des operations linéaires, Warsaw, 1932.

2. J. A. Clarkson, Uniformly convex spaces, Trans. Amer. Math. Soc. vol. 40 (1936) pp. $396-414$.

3. M. M. Day, Reflexive Banach spaces not isomorphic to uniformly convex spaces, Bull. Amer. Math. Soc. vol. 47 (1941) pp. 313-317.

4. D. Milman, On some criteria for the regularity of spaces of type (B), C. R. (Doklady) Acad. Sci. URSS. vol. 20 (1938) pp. 243-246.

5. F. J. Murray and J. v. Neumann, On rings of operators, Ann. of Math. (2) vol. 37(1936) pp. 116-229.

6. B. J. Pettis, Proof that every uniformly convex space is reflexive; Duke Math. J. vol. 5 (1939) pp. 249-253.

7. R. Schatten, On the direct product of Banach spaces, Trans. Amer. Math. Soc. vol. 53 (1943) pp. 195-217.

United States Army, Atlanta, GA. 